Supporting Information for:

\title{
Ultrafast Intersystem Crossing in 1-Nitronaphthalene. An Experimental and Computational Study
}

Jimena S. Zugazagoitia, César Xavier Almora-Díaz and Jorge Peon*

Universidad Nacional Autónoma de México, Instituto de Química

Ciudad Universitaria, 04510, México, D.F., México.

jpeon@servidor.unam.mx

Table S1. 1-Nitronaphthalene in acetonitrile. (PCM)PBE0/6-311G(d,p) ground state optimized geometry.

\begin{tabular}{|c|c|c|c|c|}
\hline \multirow{2}{*}{$\begin{array}{c}\text { Center } \\
\text { Number }\end{array}$} & Atomic & \multicolumn{3}{|c|}{ Coordinates (Angstroms) } \\
\cline { 3 - 5 } & Number & $\mathrm{X}$ & $\mathrm{Y}$ & $\mathrm{Z}$ \\
\hline 1 & 6 & -1.061092 & 0.212206 & 0.006127 \\
\hline 2 & 6 & -1.418945 & 1.538453 & 0.023981 \\
\hline 3 & 6 & -0.431564 & 2.533784 & 0.068555 \\
\hline 4 & 6 & 0.891853 & 2.175359 & 0.074429 \\
\hline 5 & 6 & 2.657935 & 0.473619 & 0.002025 \\
\hline 6 & 6 & 3.053606 & -0.835632 & -0.067961 \\
\hline 7 & 6 & 2.082437 & -1.855028 & -0.121784 \\
\hline 8 & 6 & 0.741245 & -1.562699 & -0.087640 \\
\hline 9 & 6 & 1.284195 & 0.816730 & 0.028046 \\
\hline 10 & 6 & 0.297824 & -0.219629 & -0.002560 \\
\hline 11 & 7 & -2.169156 & -0.740012 & 0.016732 \\
\hline 12 & 8 & -2.006274 & -1.818672 & 0.558655 \\
\hline 13 & 8 & -3.217850 & -0.397228 & -0.498197 \\
\hline 14 & 1 & -2.472021 & 1.804138 & 0.021634 \\
\hline 15 & 1 & -0.726429 & 3.579558 & 0.103575 \\
\hline 16 & 1 & 1.669529 & 2.936931 & 0.110281 \\
\hline 17 & 1 & 3.391481 & 1.277495 & 0.031543 \\
\hline 18 & 1 & 4.110740 & -1.091059 & -0.089865 \\
\hline 19 & 1 & 2.397810 & -2.894091 & -0.191665 \\
\hline 20 & 1 & 0.021010 & -2.368668 & -0.125595 \\
\hline
\end{tabular}


Table S2. 1-Nitronaphthalene in cyclohexane. (PCM)PBE0/6-311G(d,p) ground state optimized geometry.

\begin{tabular}{|c|c|c|c|c|}
\hline \multirow{2}{*}{$\begin{array}{c}\text { Center } \\
\text { Number }\end{array}$} & Atomic & \multicolumn{3}{|c|}{ Coordinates (Angstroms) } \\
\cline { 3 - 5 } & Number & $\mathrm{X}$ & $\mathrm{Y}$ & $\mathrm{Z}$ \\
\hline 1 & 6 & -1.059405 & 0.210969 & 0.010342 \\
\hline 2 & 6 & -1.417043 & 1.535716 & 0.030857 \\
\hline 3 & 6 & -0.430843 & 2.532177 & 0.073140 \\
\hline 4 & 6 & 0.891967 & 2.175624 & 0.072217 \\
\hline 5 & 6 & 2.658110 & 0.473567 & -0.006043 \\
\hline 6 & 6 & 3.052983 & -0.835037 & -0.071957 \\
\hline 7 & 6 & 2.082069 & -1.854246 & -0.113500 \\
\hline 8 & 6 & 0.742297 & -1.561754 & -0.075643 \\
\hline 9 & 6 & 1.285556 & 0.818182 & 0.024666 \\
\hline 10 & 6 & 0.299307 & -0.218017 & -0.000035 \\
\hline 11 & 7 & -2.173635 & -0.743105 & 0.012718 \\
\hline 12 & 8 & -2.003985 & -1.829229 & 0.533404 \\
\hline 13 & 8 & -3.221068 & -0.382903 & -0.487887 \\
\hline 14 & 1 & -2.469625 & 1.793234 & 0.021234 \\
\hline 15 & 1 & -0.725667 & 3.575713 & 0.108138 \\
\hline 16 & 1 & 1.666621 & 2.937146 & 0.103498 \\
\hline 17 & 1 & 3.392160 & 1.274281 & 0.017853 \\
\hline 18 & 1 & 4.108061 & -1.089832 & -0.097215 \\
\hline 19 & 1 & 2.396382 & -2.891944 & -0.174623 \\
\hline 20 & 1 & 0.017948 & -2.362894 & -0.096309 \\
\hline
\end{tabular}


Table S3. 1-Nitronaphthalene in the gas phase. PBE0/6-311G(d,p) ground state optimized geometry.

\begin{tabular}{|c|c|c|c|c|}
\hline \multirow{2}{*}{$\begin{array}{c}\text { Center } \\
\text { Number }\end{array}$} & Atomic & \multicolumn{3}{|c|}{ Coordinates (Angstroms) } \\
\cline { 3 - 5 } & Number & $\mathrm{X}$ & $\mathrm{Y}$ & $\mathrm{Z}$ \\
\hline 1 & 6 & -1.058699 & 0.208959 & 0.011531 \\
\hline 2 & 6 & -1.416379 & 1.532957 & 0.032719 \\
\hline 3 & 6 & -0.431454 & 2.530708 & 0.073023 \\
\hline 4 & 6 & 0.891204 & 2.176081 & 0.069700 \\
\hline 5 & 6 & 2.658406 & 0.475485 & -0.008862 \\
\hline 6 & 6 & 3.054249 & -0.832403 & -0.071534 \\
\hline 7 & 6 & 2.084434 & -1.852321 & -0.107250 \\
\hline 8 & 6 & 0.744940 & -1.561303 & -0.069043 \\
\hline 9 & 6 & 1.286050 & 0.819405 & 0.022840 \\
\hline 10 & 6 & 0.300503 & -0.217546 & 0.000918 \\
\hline 11 & 7 & -2.177425 & -0.745917 & 0.010471 \\
\hline 12 & 8 & -1.998976 & -1.842206 & 0.504080 \\
\hline 13 & 8 & -3.229059 & -0.369640 & -0.465524 \\
\hline 14 & 1 & -2.469164 & 1.784416 & 0.018919 \\
\hline 15 & 1 & -0.726766 & 3.573007 & 0.106930 \\
\hline 16 & 1 & 1.664382 & 2.937634 & 0.098356 \\
\hline 17 & 1 & 3.391492 & 1.275764 & 0.012303 \\
\hline 18 & 1 & 4.108536 & -1.085791 & -0.097483 \\
\hline 19 & 1 & 2.399533 & -2.888894 & -0.163339 \\
\hline 20 & 1 & 0.018723 & -2.360081 & -0.081688 \\
\hline
\end{tabular}


Table S4. 1-Nitronaphthalene in methanol. (PCM)PBE0/6-311G(d,p) ground state optimized geometry.

\begin{tabular}{|c|c|c|c|c|}
\hline \multirow{2}{*}{$\begin{array}{c}\text { Center } \\
\text { Number }\end{array}$} & Atomic & \multicolumn{3}{|c|}{ Coordinates (Angstroms) } \\
\cline { 3 - 5 } & Number & $\mathrm{X}$ & $\mathrm{Y}$ & $\mathrm{Z}$ \\
\hline 1 & 6 & -1.060564 & 0.214481 & 0.006166 \\
\hline 2 & 6 & -1.417870 & 1.540569 & 0.024665 \\
\hline 3 & 6 & -0.429033 & 2.534708 & 0.070651 \\
\hline 4 & 6 & 0.893946 & 2.174840 & 0.076453 \\
\hline 5 & 6 & 2.658137 & 0.470024 & 0.001754 \\
\hline 6 & 6 & 3.050848 & -0.840045 & -0.070069 \\
\hline 7 & 6 & 2.077823 & -1.857796 & -0.125035 \\
\hline 8 & 6 & 0.737324 & -1.562838 & -0.089984 \\
\hline 9 & 6 & 1.285080 & 0.815831 & 0.028418 \\
\hline 10 & 6 & 0.297354 & -0.218858 & -0.003169 \\
\hline 11 & 7 & -2.168164 & -0.738227 & 0.017379 \\
\hline 12 & 8 & -2.012091 & -1.805799 & 0.582548 \\
\hline 13 & 8 & -3.208814 & -0.405633 & -0.519773 \\
\hline 14 & 1 & -2.470858 & 1.807010 & 0.021920 \\
\hline 15 & 1 & -0.722594 & 3.580801 & 0.106783 \\
\hline 16 & 1 & 1.672306 & 2.935604 & 0.113559 \\
\hline 17 & 1 & 3.393422 & 1.272255 & 0.032380 \\
\hline 18 & 1 & 4.107439 & -1.097627 & -0.092431 \\
\hline 19 & 1 & 2.391436 & -2.897254 & -0.196501 \\
\hline 20 & 1 & 0.014952 & -2.367234 & 0.128663 \\
\hline
\end{tabular}


TABLE S5: Excitation energies (eV) and largest excitation coefficients for 1-NN in acetonitrile as computed by the (PCM)TD-PBE0/6-311++G(d,p) method. The calculation was made at a partially optimized geometry maintaining the $\mathrm{C}_{10}-\mathrm{C}_{1}$ $\mathrm{N}_{11}-\mathrm{O}_{12}$ dihedral angle at $0^{\circ}$ through the (PCM)TD-PBE0/6-311G(d,p) method.

\begin{tabular}{|c|c|c|c|c|c|c|c|}
\hline \multicolumn{5}{|c|}{ Singlet Manifold } & \multicolumn{4}{c|}{ Triplet Manifold } \\
\hline State & Transition & Coefficient & Energy & State & Transition & Coefficient & Energy \\
& & & & & & & \\
\hline 1 & $45 \rightarrow 46$ & 0.66145 & 3.3119 & 1 & $45 \rightarrow 46$ & 0.73508 & 2.2423 \\
\hline 2 & $43 \rightarrow 46$ & 0.65118 & 3.8376 & 2 & $40 \rightarrow 46$ & 0.69753 & 3.0396 \\
\hline 3 & $44 \rightarrow 46$ & 0.66169 & 3.8446 & 3 & $44 \rightarrow 46$ & 0.61560 & 3.2936 \\
\hline & & & & 4 & $43 \rightarrow 46$ & 0.68604 & 3.3085 \\
\hline & & & & 5 & $45 \rightarrow 47$ & 0.61737 & 3.6091 \\
\hline & & & & 6 & $41 \rightarrow 46$ & 0.66338 & 4.0024 \\
\hline & & & & 7 & $42 \rightarrow 46$ & 0.55215 & 4.1321 \\
\hline
\end{tabular}


TABLE S6: Excitation energies (eV) and largest excitation coefficients for 1-NN in acetonitrile as computed by the (PCM)TD-PBE0/6-311++G(d,p) method. The calculation was made at a partially optimized geometry maintaining the $\mathrm{C}_{10}-\mathrm{C}_{\mathbf{1}^{-}}$$\mathrm{N}_{11}-\mathrm{O}_{12}$ dihedral angle at $15^{\circ}$ through the (PCM)TD-PBE0/6-311G(d,p) method.

\begin{tabular}{|c|c|c|c|c|c|c|c|}
\hline \multicolumn{5}{|c|}{ Singlet Manifold } & \multicolumn{4}{c|}{ Triplet Manifold } \\
\hline State & Transition & Coefficient & Energy & State & Transition & Coefficient & Energy \\
& & & & & & & \\
\hline 1 & $45 \rightarrow 46$ & 0.66293 & 3.3221 & 1 & $45 \rightarrow 46$ & 0.72574 & 2.2728 \\
\hline 2 & $44 \rightarrow 46$ & 0.53928 & 3.8685 & 2 & $40 \rightarrow 46$ & 0.71744 & 3.0605 \\
& $43 \rightarrow 46$ & -0.33811 & & & & & \\
\hline 3 & $43 \rightarrow 46$ & 0.42321 & 3.8887 & 3 & $44 \rightarrow 46$ & 0.47882 & 3.2987 \\
& $44 \rightarrow 46$ & 0.39247 & & & $43 \rightarrow 46$ & 0.40016 & \\
& $42 \rightarrow 46$ & 0.30627 & & & & & \\
\hline & & & & 4 & $44 \rightarrow 46$ & 0.43679 & 3.3501 \\
& & & & & $43 \rightarrow 46$ & -0.41163 & \\
& & & & 5 & $45 \rightarrow 47$ & 0.59588 & 3.5930 \\
\hline & & & & 6 & $41 \rightarrow 46$ & 0.63148 & 4.0206 \\
\hline & & & & 7 & $42 \rightarrow 46$ & 0.44300 & 4.1731 \\
& & & & & $44 \rightarrow 48$ & -0.31325 & \\
& & & & & $43 \rightarrow 46$ & -0.30794 & \\
\hline
\end{tabular}


TABLE S7: Excitation energies (eV) and largest excitation coefficients for 1-NN in acetonitrile as computed by the (PCM)TD-PBE0/6-311++G(d,p) method. The calculation was made at a partially optimized geometry maintaining the $\mathrm{C}_{10}-\mathrm{C}_{1}$ $\mathrm{N}_{11}-\mathrm{O}_{12}$ dihedral angle at $45^{\circ}$ through the (PCM)TD-PBE0/6-311G(d,p) method.

\begin{tabular}{|c|c|c|c|c|c|c|c|}
\hline \multicolumn{3}{|c|}{ Singlet Manifold } & \multicolumn{4}{c|}{ Triplet Manifold } \\
\hline State & Transition & Coefficient & Energy & State & Transition & Coefficient & Energy \\
& & & & & & & \\
\hline 1 & $45 \rightarrow 46$ & 0.66241 & 3.3034 & 1 & $45 \rightarrow 46$ & 0.66495 & 2.3851 \\
\hline 2 & $44 \rightarrow 46$ & 0.66961 & 3.9865 & 2 & $40 \rightarrow 46$ & 0.80800 & 3.0799 \\
\hline 3 & $43 \rightarrow 46$ & 0.54250 & 4.0598 & 3 & $45 \rightarrow 47$ & 0.54268 & 3.2475 \\
& & & & & $43 \rightarrow 46$ & 0.35111 & \\
\hline & & & & 4 & $44 \rightarrow 46$ & 0.65959 & 3.5314 \\
\hline & & & & 5 & $43 \rightarrow 46$ & 0.37618 & 3.6473 \\
& & & & & $45 \rightarrow 47$ & -0.30551 & \\
& & & & & $45 \rightarrow 46$ & -0.27044 & \\
& & & & & $41 \rightarrow 46$ & 0.27035 & \\
\hline & & & & 6 & $42 \rightarrow 46$ & -0.22969 & \\
\hline & & & & & $41 \rightarrow 46$ & 0.44198 & 4.0485 \\
& & & & 7 & $45 \rightarrow 48$ & 0.69032 & 4.2106 \\
\hline
\end{tabular}


TABLE S8: Excitation energies (eV) and largest excitation coefficients for 1-NN in cyclohexane as computed by the $(\mathrm{PCM}) \mathrm{TD}-\mathrm{PBE} 0 / 6-311++\mathrm{G}(\mathrm{d}, \mathrm{p})$ method. The calculation was made at a partially optimized geometry maintaining the $\mathrm{C}_{10}-\mathrm{C}_{1}$ $\mathrm{N}_{11}-\mathrm{O}_{12}$ dihedral angle at $0^{\circ}$ through the (PCM)TD-PBE0/6-311G(d,p) method.

\begin{tabular}{|c|c|c|c|c|c|c|c|}
\hline \multicolumn{4}{|c|}{ Singlet Manifold } & \multicolumn{4}{c|}{ Triplet Manifold } \\
\hline State & Transition & Coefficient & Energy & State & Transition & Coefficient & Energy \\
& & & & & & & \\
\hline 1 & $45 \rightarrow 46$ & 0.66398 & 3.4521 & 1 & $45 \rightarrow 46$ & 0.73258 & 2.3095 \\
\hline 2 & $43 \rightarrow 46$ & 0.65344 & 3.8078 & 2 & $40 \rightarrow 46$ & 0.74493 & 2.9574 \\
\hline 3 & $44 \rightarrow 46$ & 0.65747 & 3.9969 & 3 & $43 \rightarrow 46$ & 0.68082 & 3.2704 \\
\hline & & & & 4 & $44 \rightarrow 46$ & 0.66864 & 3.4049 \\
\hline & & & & 5 & $45 \rightarrow 47$ & 0.58184 & 3.7035 \\
\hline & & & & 6 & $41 \rightarrow 46$ & 0.67208 & 3.9120 \\
\hline & & & & 7 & $42 \rightarrow 46$ & 0.46233 & 4.2027 \\
& & & & & $44 \rightarrow 48$ & -0.37329 & \\
& & & & & $45 \rightarrow 48$ & -0.31863 & \\
\hline
\end{tabular}


TABLE S9: Excitation energies (eV) and largest excitation coefficients for 1-NN in cyclohexane as computed by the (PCM)TD-PBE0/6-311++G(d,p) method. The calculation was made at a partially optimized geometry maintaining the $\mathrm{C}_{10}-\mathrm{C}_{1^{-}}$$\mathrm{N}_{11}-\mathrm{O}_{12}$ dihedral angle at $15^{\circ}$ through the (PCM)TD-PBE0/6-311 G(d,p) method.

\begin{tabular}{|c|c|c|c|c|c|c|c|}
\hline \multicolumn{5}{|c|}{ Singlet Manifold } & \multicolumn{4}{c|}{ Triplet Manifold } \\
\hline State & Transition & Coefficient & Energy & State & Transition & Coefficient & Energy \\
& & & & & & & \\
\hline 1 & $45 \rightarrow 46$ & 0.66304 & 3.4658 & 1 & $45 \rightarrow 46$ & 0.72489 & 2.3339 \\
\hline 2 & $43 \rightarrow 46$ & 0.61577 & 3.8513 & 2 & $40 \rightarrow 46$ & 0.74083 & 2.9698 \\
\hline 3 & $44 \rightarrow 46$ & 0.65424 & 4.0260 & 3 & $43 \rightarrow 46$ & 0.63708 & 3.2889 \\
\hline & & & & 4 & $44 \rightarrow 46$ & 0.66357 & 3.4395 \\
\hline & & & & 5 & $45 \rightarrow 47$ & 0.56656 & 3.6961 \\
\hline & & & & 6 & $41 \rightarrow 46$ & 0.64340 & 3.9331 \\
\hline & & & & 7 & $45 \rightarrow 48$ & 0.43516 & 4.2243 \\
& & & & & $42 \rightarrow 46$ & 0.38897 & \\
& & & & & $44 \rightarrow 48$ & -0.31136 & \\
\hline
\end{tabular}


TABLE S10: Excitation energies (eV) and largest excitation coefficients for 1-NN in cyclohexane as computed by the (PCM)TD-PBE0/6-311++G(d,p) method. The calculation was made at a partially optimized geometry maintaining the $\mathrm{C}_{10}-\mathrm{C}_{\mathbf{1}^{-}}$$\mathrm{N}_{11}-\mathrm{O}_{12}$ dihedral angle at $45^{\circ}$ through the (PCM)TD-PBE0/6-311G(d,p) method.

\begin{tabular}{|c|c|c|c|c|c|c|c|}
\hline \multicolumn{3}{|c|}{ Singlet Manifold } & \multicolumn{4}{c|}{ Triplet Manifold } \\
\hline State & Transition & Coefficient & Energy & State & Transition & Coefficient & Energy \\
& & & & & & & \\
\hline 1 & $45 \rightarrow 46$ & 0.64724 & 3.4770 & 1 & $45 \rightarrow 46$ & 0.67256 & 2.4276 \\
\hline 2 & $43 \rightarrow 46$ & 0.56345 & 4.0604 & 2 & $40 \rightarrow 46$ & 0.76083 & 2.9976 \\
\hline 3 & $44 \rightarrow 46$ & 0.64325 & 4.1539 & 3 & $43 \rightarrow 46$ & 0.47401 & 3.2861 \\
& & & & & $45 \rightarrow 47$ & -0.45800 & \\
\hline & & & & 4 & $44 \rightarrow 46$ & 0.60960 & 3.6310 \\
\hline & & & & 5 & $45 \rightarrow 47$ & 0.36524 & 3.7477 \\
& & & & & $44 \rightarrow 46$ & 0.27500 & \\
& & & & & $45 \rightarrow 46$ & -0.27226 & \\
& & & & & $43 \rightarrow 46$ & 0.25278 & \\
& & & & & $41 \rightarrow 46$ & -0.21412 & \\
\hline & & & & 6 & $42 \rightarrow 46$ & 0.57003 & 3.9901 \\
\hline & & & & 7 & $45 \rightarrow 48$ & 0.70528 & 4.2177 \\
\hline
\end{tabular}


Figure S1. Frontier DFT orbitals of 1-nitronaphthalene in methanol solution as calculated by the (PCM)PBE0/6-311++G(d,p) method at the (PCM)PBE0/6$311 G(d, p)$ optimized geometry for the electronic ground state. Isosurface value: 0.03. In brackets we include the orbital energy $(\mathrm{eV})$.

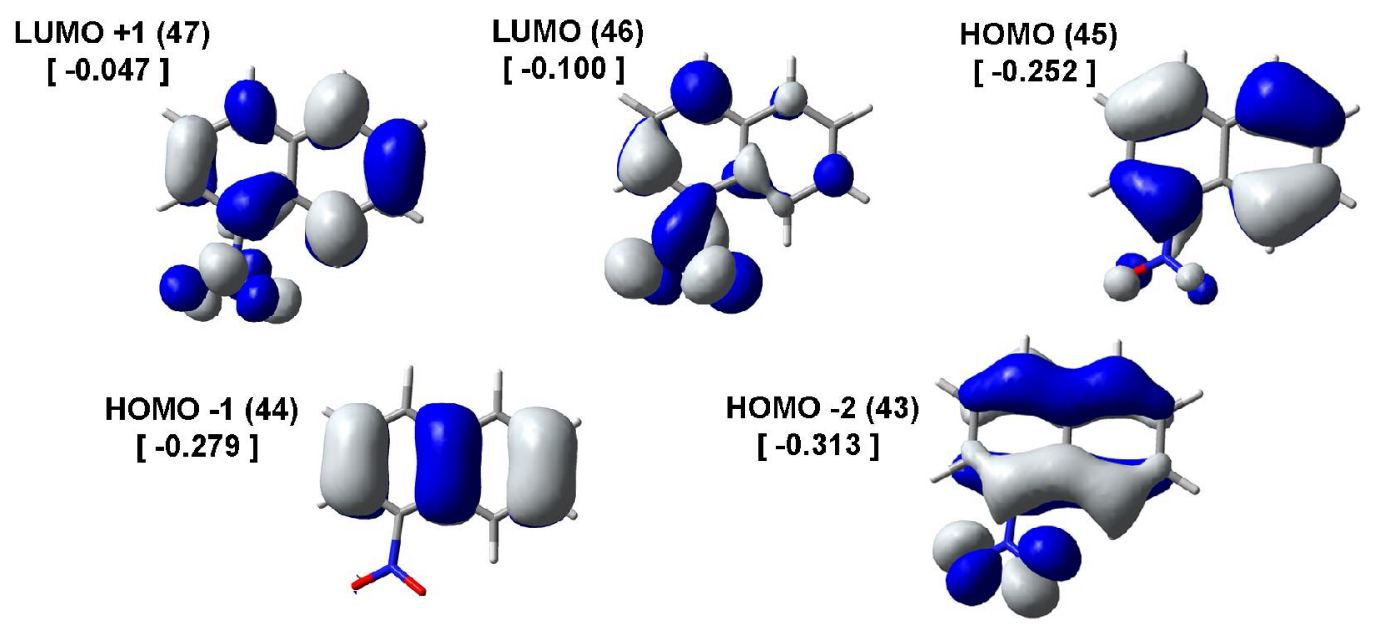


Figure S2. Frontier DFT orbitals of 1-nitronaphthalene in cyclohexane solution as calculated by the (PCM)PBE0/6-311++G(d,p) method at the (PCM) PBE0/6$311 G(d, p)$ optimized geometry for the electronic ground state. Isosurface value: 0.03. In brackets we include the orbital energy $(\mathrm{eV})$.

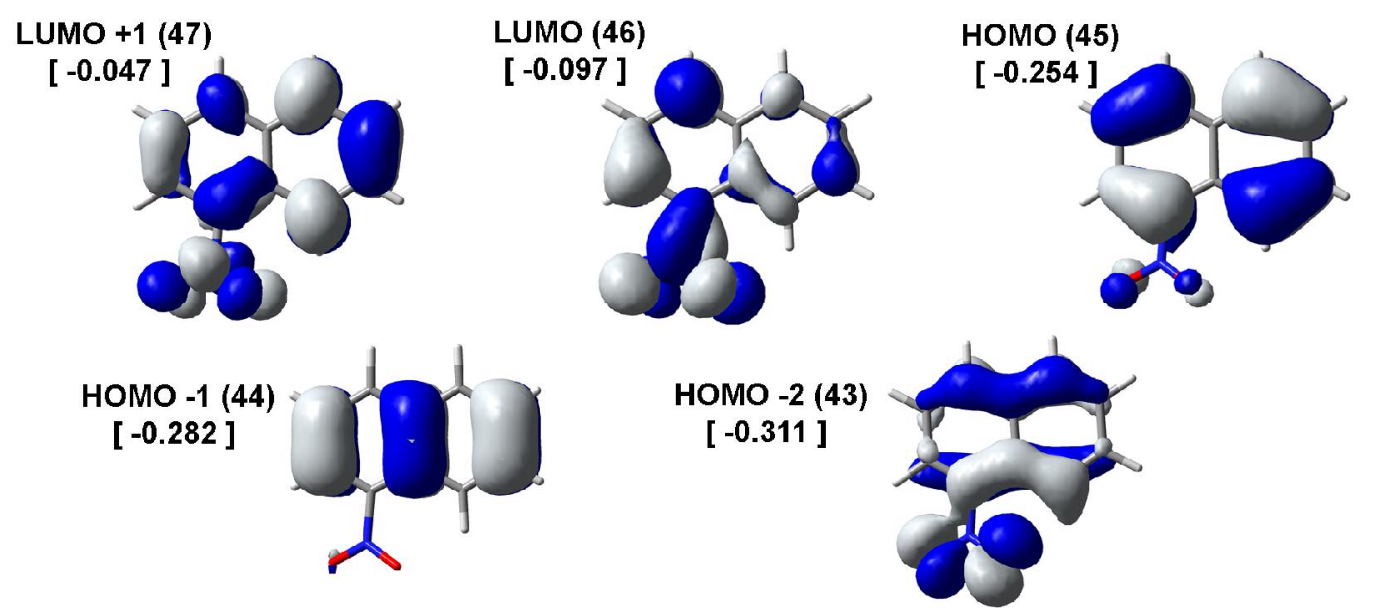


Figure S3. Frontier DFT orbitals of 1-nitronaphthalene in the gas phase as calculated by the PBE0/6-311++G(d,p) method at the PBE0/6-311G(d,p) optimized geometry for the electronic ground state. Isosurface value: 0.03 . In brackets we include the orbital energy (eV).

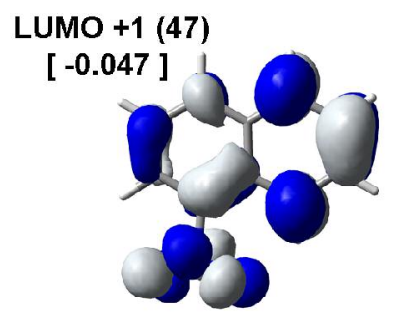

HOMO -1 (44) [ -0.285$]$

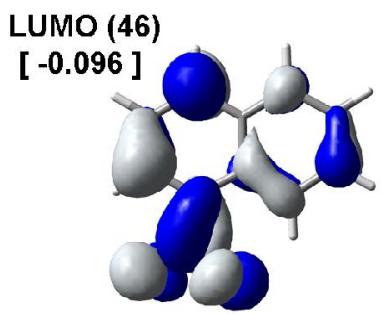

HOMO (45)

$[-0.257]$

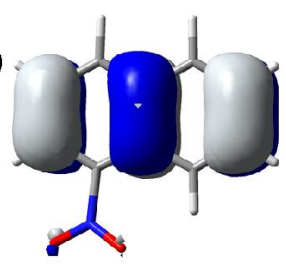

HOMO -2 (43) $[-0.310]$

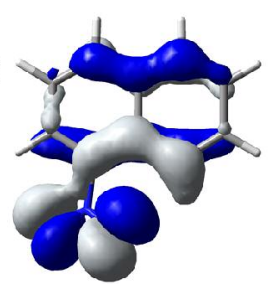

